M. Nazarianpoor · J. M. Rassias · Gh. Sadeghi

\title{
Stability and nonstability of octadecic functional equation in multi-normed spaces
}

Received: 11 March 2017 / Accepted: 19 September 2017 / Published online: 30 September 2017

(C) The Author(s) 2017. This article is an open access publication

Abstract In this paper, we introduce octadecic functional equation. Moreover, we prove the stability of the octadecic functional equation in multi-normed spaces by using the fixed point method.

Mathematics Subject Classification $39 \mathrm{~A} 11 \cdot 39 \mathrm{~B} 52$

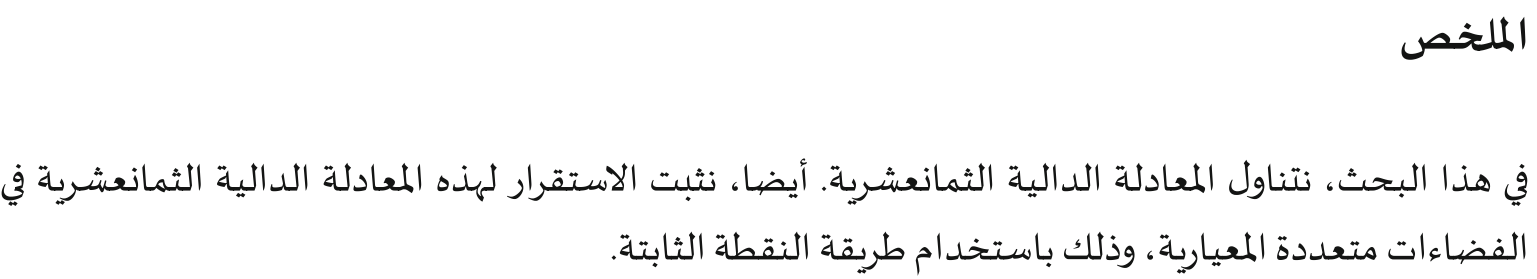

\section{Introduction}

In 1940, Ulam [17] proposed the following question concerning the stability of group homomorphisms:

Let $G_{1}$ be a group and $\left(G_{2}, d\right)$ be a metric group. Given $\varepsilon>0$, does there exist a $\delta>0$, such that if a mapping $h: G_{1} \rightarrow G_{2}$ satisfies the inequality $d(h(x y), h(x) h(y))<\delta$ for all $x, y \in G_{1}$, then there exists a homomorphism $H: G_{1} \rightarrow G_{2}$, such that $d(h(x), H(x))<\varepsilon$ for all $x \in G_{1}$ ?

In the next year, 1941, Hyers [8] solved the famous stability problem of Ulam in Banach spaces:

Let $X$ be a normed space and $Y$ be a Banach space. Suppose that for some $\varepsilon>0$, the mapping $f: X \rightarrow Y$ satisfies $\|f(x+y)-f(x)-f(y)\| \leq \varepsilon$ for all $x, y \in X$. Then there exists a unique additive mapping $T: X \rightarrow Y$, such that $\|f(x)-T(x)\| \leq \varepsilon$ for all $x \in X$.

In 1978, Rassias [15] proved the following theorem:

M. Nazarianpoor · Gh. Sadeghi $(\bowtie)$

Department of Mathematics and Computer Sciences, Hakim Sabzevari University, P.O. Box 397, Sabzevar, Iran

E-mail: ghadir54@gmail.com; g.sadeghi@hsu.ac.ir

M. Nazarianpoor

E-mail: mehdi.nazarianpoor@yahoo.com; m.nazarianpoor@hsu.ac.ir

J. M. Rassias

Pedagogical Department E. E, Section of Mathematics and Informatics, National and Capodistrian University of Athens, Athens, Greece

E-mail: jrassias@primedu.uoa.gr; Ioannis.Rassias@primedu.uoa.gr 
Let $X$ and $Y$ be real normed spaces with $Y$ complete. Let $f: X \rightarrow Y$ be a mapping such that, for each fixed $x \in X$, the mapping $h(t)=f(t x)$ is continuous on $\mathbb{R}$, and let $\varepsilon \geq 0$ and $p \in[0,1)$ be such that

$$
\|f(x+y)-f(x)-f(y)\| \leq \varepsilon\left(\|x\|^{p}+\|y\|^{p}\right)
$$

holds for all $x, y \in X$. Then there exists a unique linear mapping $T: X \rightarrow Y$, such that

$$
\|f(x)-T(x)\| \leq \varepsilon \frac{\|x\|^{p}}{1-2^{p-1}}
$$

for all $x \in X$. Since the past few decades several stability problems of functional equations have been investigated $[1,2,5-7,11,12,14,19]$. Xu et al. [20] proved the general solution and the stability of the quintic functional equation

$$
f(x+3 y)-5 f(x+2 y)+10 f(x+y)-10 f(x)+5 f(x-y)-f(x-2 y)=120 f(y)
$$

and the sextic functional equation

$$
\begin{aligned}
& f(x+3 y)-6 f(x+2 y)+15 f(x+y)-20 f(x)+15 f(x-y)-6 f(x-2 y) \\
& \quad+f(x-3 y)=720 f(y)
\end{aligned}
$$

in quasi- $\beta$-normed spaces.

The general solution and the stability of the septic functional equation

$$
\begin{aligned}
& f(x+4 y)-7 f(x+3 y)+21 f(x+2 y)-35 f(x+y)-35 f(x)-21 f(x-y) \\
& \quad+7 f(x-2 y)-f(x-3 y)=5040 f(y)
\end{aligned}
$$

and the octic functional equation

$$
\begin{aligned}
& f(x+4 y)-8 f(x+3 y)+28 f(x+2 y)-56 f(x+y)-70 f(x)-56 f(x-y) \\
& \quad+28 f(x-2 y)-8 f(x-3 y)+f(x-4 y)=40320 f(y)
\end{aligned}
$$

in quasi- $\beta$-normed spaces were investigated by Xu and Rassias [18]. Rassias and Eslamian [13] investigated the general solution of a nonic functional equation

$$
\begin{aligned}
& f(x+5 y)-9 f(x+4 y)+36 f(x+3 y)-84 f(x+2 y)-126 f(x+y)-126 f(x) \\
& \quad+84 f(x-y)-36 f(x-2 y)+9 f(x-3 y)-f(x-4 y)=9 ! f(y)
\end{aligned}
$$

and proved the stability of nonic functional equation in quasi- $\beta$-normed spaces by using the fixed point method. A fixed point approach for the stability of decic functional equation

$$
\begin{aligned}
& f(x+5 y)-10 f(x+4 y)+45 f(x+3 y)-120 f(x+2 y)-210 f(x+y)-252 f(x) \\
& \quad+210 f(x-y)-120 f(x-2 y)+45 f(x-3 y)-10 f(x-4 y)+f(x-5 y)=10 ! f(y)
\end{aligned}
$$

in quasi- $\beta$-normed spaces was investigated by Ravi et al. [16].

Let $(X,\|\cdot\|)$ be a complex normed space, and $k \in \mathbb{N}$. We denote the group of permutations on $k$ symbols by $\mathfrak{G}_{k}$.

Definition 1.1 $[3,4,9]$ A multi-norm on $\left\{X^{k}: k \in \mathbb{N}\right\}$ is a sequence

$$
\left(\|\cdot\|_{k}\right)=\left(\|\cdot\|_{k}: k \in \mathbb{N}\right)
$$

such that $\|\cdot\|_{k}$ is a norm on $X^{k}$ for each $k \in \mathbb{N},\|x\|_{1}=\|x\|$ for each $x \in X$, and the following axioms are satisfied for each $k \in \mathbb{N}$ with $k \geq 2$ :

(MN1)

$$
\left\|x_{\sigma(1)}, \ldots, x_{\sigma(k)}\right\|_{k}=\left\|x_{1}, \ldots, x_{k}\right\|_{k} \quad\left(\sigma \in \mathfrak{G}_{k}, x_{1}, \ldots, x_{k} \in X\right)
$$


(MN2)

$$
\begin{aligned}
& \left\|\alpha_{1} x_{1}, \ldots, \alpha_{k} x_{k}\right\|_{k} \leq\left(\max _{i \in \mathbb{N}_{k}}\left|\alpha_{i}\right|\right)\left\|x_{1}, \ldots, x_{k}\right\|_{k} \\
& \left(\alpha_{1}, \ldots, \alpha_{k} \in \mathbb{C}, x_{1}, \ldots, x_{k} \in X\right)
\end{aligned}
$$

(MN3)

$$
\left\|x_{1}, \ldots, x_{k-1}, 0\right\|_{k}=\left\|x_{1}, \ldots, x_{k-1}\right\|_{k-1} \quad\left(x_{1}, \ldots, x_{k-1} \in X\right)
$$

(MN4)

$$
\left\|x_{1}, \ldots, x_{k-1}, x_{k-1}\right\|_{k}=\left\|x_{1}, \ldots, x_{k-1}\right\|_{k-1} \quad\left(x_{1}, \ldots, x_{k-1} \in X\right) .
$$

In this case, we say that $\left(\left(X^{k},\|\cdot\|_{k}\right): k \in \mathbb{N}\right)$ is a multi-normed space. If $(X,\|\|$.$) is a Banach space, then$ $\left(X^{k},\|\cdot\|_{k}\right)$ is a Banach space for each $k \in \mathbb{N}$, in this case $\left(\left(X^{k},\|\cdot\|_{k}\right): k \in \mathbb{N}\right)$ is a multi-Banach space.

Example 1.2 Let $(X,\|\|$.$) be a Banach lattice, and let us define$

$$
\left\|x_{1}, \ldots, x_{k}\right\|_{k}:=\left\|\left|x_{1}\right| \vee \cdots \vee\left|x_{k}\right|\right\| \quad\left(x_{1}, \ldots, x_{k} \in X\right) .
$$

Then $\left(\left(X^{k},\|\cdot\|_{k}\right): k \in \mathbb{N}\right)$ is a multi-Banach space.

Let $X$ and $Y$ be real vector spaces and $f: X \rightarrow Y$ be a mapping. We define a mapping Df : $X^{2} \rightarrow Y$ by

$$
\begin{aligned}
\operatorname{Df}(x, y): & =f(x+9 y)-18 f(x+8 y)+153 f(x+7 y)-816 f(x+6 y)+3060 f(x+5 y) \\
& -8568 f(x+4 y)+18564 f(x+3 y)-31824 f(x+2 y)+43758 f(x+y)-48620 f(x) \\
& +43758 f(x-y)-31824 f(x-2 y)+18564 f(x-3 y)-8568 f(x-4 y) \\
& +3060 f(x-5 y)-816 f(x-6 y)+153 f(x-7 y)-18 f(x-8 y)+f(x-9 y) \\
& -18 ! f(y),
\end{aligned}
$$

for all $x, y \in X$, where $18 !=6402373705728000$.

In this paper, we introduce the following octadecic functional equation:

$$
\operatorname{Df}(x, y)=0,
$$

for all $x, y \in X$. Moreover, we prove the stability of the octadecic functional equation (1.1) in multi-normed spaces by using the standard fixed point method:

Theorem 1.3 [10] If $X$ and $Y$ are real vector spaces and $f: X \rightarrow Y$ is a mapping satisfying octadecic functional equation (1.1) for all $x, y \in X$, then $f$ is an octadecic mapping, i.e., $f(x)=x^{18}$.

\section{Stability of the functional equation (1.1) in multi-normed spaces}

In this section, we prove the generalized Hyers-Ulam stability of the octadecic functional equation (1.1) in multi-normed spaces. Throughout this section, we assume that $X$ is a normed space and that $Y$ is a Banach space. Let $\left(\left(Y^{k},\|\cdot\|_{k}\right): k \in \mathbb{N}\right)$ be a multi-Banach space.

Theorem 2.1 [10] Let $\phi: X^{2} \rightarrow[0, \infty)$ be a mapping, such that there exists $L<1$ with $\phi(2 x, 2 y) \leq$ $2^{18} L \phi(x, y)$ for all $x, y \in X$. Let $f: X \rightarrow Y$ be a mapping satisfying

$$
\|\operatorname{Df}(x, y)\| \leq \phi(x, y),
$$

for all $x, y \in X$. Then there exists a unique octadecic mapping $Q: X \rightarrow Y$, such that

$$
\|f(x)-Q(x)\| \leq \frac{1}{2^{18}(1-L)} \psi(x),
$$

for all $x \in X$, where

$$
\psi(x):=(3201186852864000)^{-1}(24310 \phi(0, x)+43758 \phi(x, x)+31824 \phi(2 x, x)
$$




$$
\begin{aligned}
& +18564 \phi(3 x, x)+8568 \phi(4 x, x)+3060 \phi(5 x, x)+816 \phi(6 x, x)+153 \phi(7 x, x) \\
& +18 \phi(8 x, x)+\phi(9 x, x)+\frac{\phi(0,2 x)}{2} \\
& +\left(\frac{4537567325}{6402373705728000}+\frac{48886173}{12705510619017216000}\right) \phi(0,0) \\
& +\left(\frac{3234988548}{6402373705728000}+\frac{1}{2324754432000}+\frac{1}{355687428096000}\right)(\phi(x, x)+\phi(x,-x)) \\
& +\left(\frac{1922913180}{6402373705728000}+\frac{1}{41845579776000}+\frac{1}{292626432000}\right)(\phi(2 x, 2 x) \\
& +\phi(2 x,-2 x))+\frac{940105848}{6402373705728000}(\phi(3 x, 3 x)+\phi(3 x,-3 x)) \\
& +\left(\frac{371153520}{6402373705728000}+\frac{1}{402361344000}\right)(\phi(4 x, 4 x)+\phi(4 x,-4 x)) \\
& +\frac{115306920}{6402373705728000}(\phi(5 x, 5 x)+\phi(5 x,-5 x)) \\
& +\left(\frac{27123330}{6402373705728000}+\frac{1}{689762304000}\right)(\phi(6 x, 6 x)+\phi(6 x,-6 x)) \\
& +\frac{4538898}{6402373705728000}(\phi(7 x, 7 x)+\phi(7 x,-7 x)) \\
& +\left(\frac{481338}{6402373705728000}+\frac{1}{1494484992000}\right)(\phi(8 x, 8 x)+\phi(8 x,-8 x)) \\
& +\frac{24310(\phi(9 x, 9 x)+\phi(9 x,-9 x))}{6402373705728000}+\frac{\phi(10 x, 10 x)+\phi(10 x,-10 x)}{4184557977600} \\
& +\frac{\phi(12 x, 12 x)+\phi(12 x,-12 x)}{15692092416000}+\frac{\phi(14 x, 14 x)+\phi(14 x,-14 x)}{83691159552000} \\
& +\frac{\phi(16 x, 16 x)+\phi(16 x,-16 x)}{711374856192000} \\
& \left.+(12804747411456000)^{-1}(\phi(18 x, 18 x)+\phi(18 x,-18 x))\right) .
\end{aligned}
$$

Theorem 2.2 Let $k \in \mathbb{N}$ and $\phi: X^{2 k} \rightarrow[0, \infty)$ be a mapping, such that there exists $L<1$ with $\phi\left(2 x_{1}, \ldots, 2 x_{k}, 2 y_{1}, \ldots, 2 y_{k}\right) \leq 2^{18} L \phi\left(x_{1}, \ldots, x_{k}, y_{1}, \ldots, y_{k}\right)$ for all $x_{1}, \ldots, x_{k}, y_{1}, \ldots, y_{k} \in X$. Let $f: X \rightarrow Y$ be a mapping satisfying

$$
\left\|\operatorname{Df}\left(x_{1}, y_{1}\right), \ldots, \operatorname{Df}\left(x_{k}, y_{k}\right)\right\|_{k} \leq \phi\left(x_{1}, \ldots, x_{k}, y_{1}, \ldots, y_{k}\right),
$$

for all $x_{1}, \ldots, x_{k}, y_{1}, \ldots, y_{k} \in X$. Then there exists a unique octadecic mapping $Q: X \rightarrow Y$, such that

$$
\left\|f\left(x_{1}\right)-Q\left(x_{1}\right), \ldots, f\left(x_{k}\right)-Q\left(x_{k}\right)\right\|_{k} \leq \frac{1}{2^{18}(1-L)} \psi\left(x_{1}, \ldots, x_{k}\right),
$$

for all $x_{1}, \ldots, x_{k} \in X$, where

$$
\begin{aligned}
\psi\left(x_{1}, \ldots, x_{k}\right):= & (3201186852864000)^{-1}\left(24310 \phi\left(0, \ldots, 0, x_{1}, \ldots, x_{k}\right)\right. \\
& +43758 \phi\left(x_{1}, \ldots, x_{k}, x_{1}, \ldots, x_{k}\right)+31824 \phi\left(2 x_{1}, \ldots, 2 x_{k}, x_{1}, \ldots, x_{k}\right) \\
& +18564 \phi\left(3 x_{1}, \ldots, 3 x_{k}, x_{1}, \ldots, x_{k}\right)+8568 \phi\left(4 x_{1}, \ldots, 4 x_{k}, x_{1}, \ldots, x_{k}\right) \\
& +3060 \phi\left(5 x_{1}, \ldots, 5 x_{k}, x_{1}, \ldots, x_{k}\right)+816 \phi\left(6 x_{1}, \ldots, 6 x_{k}, x_{1}, \ldots, x_{k}\right) \\
& +153 \phi\left(7 x_{1}, \ldots, 7 x_{k}, x_{1}, \ldots, x_{k}\right)+18 \phi\left(8 x_{1}, \ldots, 8 x_{k}, x_{1}, \ldots, x_{k}\right) \\
& +\phi\left(9 x_{1}, \ldots, 9 x_{k}, x_{1}, \ldots, x_{k}\right)+\frac{\phi\left(0, \ldots, 0,2 x_{1}, \ldots, 2 x_{k}\right)}{2} \\
& +\left(\frac{4537567325}{6402373705728000}+\frac{48886173}{12705510619017216000}\right) \phi(0, \ldots, 0,0, \ldots, 0) \\
& +\left(\frac{3234988548}{6402373705728000}+\frac{1}{2324754432000}+\frac{1}{355687428096000}\right)
\end{aligned}
$$




$$
\begin{aligned}
& \times\left(\phi\left(x_{1}, \ldots, x_{k}, x_{1}, \ldots, x_{k}\right)+\phi\left(x_{1}, \ldots, x_{k},-x_{1}, \ldots,-x_{k}\right)\right) \\
& +\left(\frac{1922913180}{6402373705728000}+\frac{1}{41845579776000}+\frac{1}{292626432000}\right) \\
& \times\left(\phi\left(2 x_{1}, \ldots, 2 x_{k}, 2 x_{1}, \ldots, 2 x_{k}\right)+\phi\left(2 x_{1}, \ldots, 2 x_{k},-2 x_{1}, \ldots,-2 x_{k}\right)\right) \\
& +\frac{940105848}{6402373705728000}\left(\phi\left(3 x_{1}, \ldots, 3 x_{k}, 3 x_{1}, \ldots, 3 x_{k}\right)\right. \\
& \left.+\phi\left(3 x_{1}, \ldots, 3 x_{k},-3 x_{1}, \ldots,-3 x_{k}\right)\right)+\left(\frac{371153520}{6402373705728000}+\frac{1}{402361344000}\right) \\
& \times\left(\phi\left(4 x_{1}, \ldots, 4 x_{k}, 4 x_{1}, \ldots, 4 x_{k}\right)+\phi\left(4 x_{1}, \ldots, 4 x_{k},-4 x_{1}, \ldots,-4 x_{k}\right)\right) \\
& +\frac{115306920}{6402373705728000}\left(\phi\left(5 x_{1}, \ldots, 5 x_{k}, 5 x_{1}, \ldots, 5 x_{k}\right)\right. \\
& \left.+\phi\left(5 x_{1}, \ldots, 5 x_{k},-5 x_{1}, \ldots,-5 x_{k}\right)\right)+\left(\frac{27123330}{6402373705728000}+\frac{1}{689762304000}\right) \\
& \times\left(\phi\left(6 x_{1}, \ldots, 6 x_{k}, 6 x_{1}, \ldots, 6 x_{k}\right)+\phi\left(6 x_{1}, \ldots, 6 x_{k},-6 x_{1}, \ldots,-6 x_{k}\right)\right) \\
& +\frac{4538898}{6402373705728000}\left(\phi\left(7 x_{1}, \ldots, 7 x_{k}, 7 x_{1}, \ldots, 7 x_{k}\right)\right. \\
& \left.+\phi\left(7 x_{1}, \ldots, 7 x_{k},-7 x_{1}, \ldots,-7 x_{k}\right)\right)+\left(\frac{481338}{6402373705728000}+\frac{1}{1494484992000}\right) \\
& \times\left(\phi\left(8 x_{1}, \ldots, 8 x_{k}, 8 x_{1}, \ldots, 8 x_{k}\right)+\phi\left(8 x_{1}, \ldots, 8 x_{k},-8 x_{1}, \ldots,-8 x_{k}\right)\right) \\
& +\frac{24310\left(\phi\left(9 x_{1}, \ldots, 9 x_{k}, 9 x_{1}, \ldots, 9 x_{k}\right)+\phi\left(9 x_{1}, \ldots, 9 x_{k},-9 x_{1}, \ldots,-9 x_{k}\right)\right)}{6402373705728000} \\
& +\frac{\phi\left(10 x_{1}, \ldots, 10 x_{k}, 10 x_{1}, \ldots, 10 x_{k}\right)+\phi\left(10 x_{1}, \ldots, 10 x_{k},-10 x_{1}, \ldots,-10 x_{k}\right)}{4184557977600} \\
& +\frac{\phi\left(12 x_{1}, \ldots, 12 x_{k}, 12 x_{1}, \ldots, 12 x_{k}\right)+\phi\left(12 x_{1}, \ldots, 12 x_{k},-12 x_{1}, \ldots,-12 x_{k}\right)}{15692092416000} \\
& +\frac{\phi\left(14 x_{1}, \ldots, 14 x_{k}, 14 x_{1}, \ldots, 14 x_{k}\right)+\phi\left(14 x_{1}, \ldots, 14 x_{k},-14 x_{1}, \ldots,-14 x_{k}\right)}{83691159552000} \\
& +\frac{\phi\left(16 x_{1}, \ldots, 16 x_{k}, 16 x_{1}, \ldots, 16 x_{k}\right)+\phi\left(16 x_{1}, \ldots, 16 x_{k},-16 x_{1}, \ldots,-16 x_{k}\right)}{711374856192000} \\
& +(12804747411456000)^{-1}\left(\phi\left(18 x_{1}, \ldots, 18 x_{k}, 18 x_{1}, \ldots, 18 x_{k}\right)\right. \\
& \left.\left.+\phi\left(18 x_{1}, \ldots, 18 x_{k},-18 x_{1}, \ldots,-18 x_{k}\right)\right)\right) \text {. }
\end{aligned}
$$

Proof The proof is similar to the proof of Theorem 2.1.

From Theorem 2.2, we get the following corollaries:

Corollary 2.3 Let $k \in \mathbb{N}$ and $\alpha, L$ be positive real numbers, such that $\frac{1}{2^{18}} \leq L<1$. Let $f: X \rightarrow Y$ be a mapping satisfying

$$
\left\|\operatorname{Df}\left(x_{1}, y_{1}\right), \ldots, \operatorname{Df}\left(x_{k}, y_{k}\right)\right\|_{k} \leq \alpha,
$$

for all $x_{1}, \ldots, x_{k}, y_{1}, \ldots, y_{k} \in X$. Then there exists a unique octadecic mapping $Q: X \rightarrow Y$, such that

$$
\left\|f\left(x_{1}\right)-Q\left(x_{1}\right), \ldots, f\left(x_{k}\right)-Q\left(x_{k}\right)\right\|_{k} \leq \frac{1}{2^{18}(1-L)} \beta,
$$

for all $x_{1}, \ldots, x_{k} \in X$, where

$$
\begin{aligned}
\beta: & =(3201186852864000)^{-1} \alpha(24310+43758+31824+18564+8568+3060+816 \\
& +153+18+1+\frac{1}{2}+\frac{4537567325}{6402373705728000}+\frac{48886173}{12705510619017216000} \\
& +2\left(\frac{3234988548}{6402373705728000}+\frac{1}{2324754432000}+\frac{1}{355687428096000}\right)
\end{aligned}
$$




$$
\begin{aligned}
& +2\left(\frac{1922913180}{6402373705728000}+\frac{1}{41845579776000}+\frac{1}{292626432000}\right) \\
& +\frac{18802116960}{6402373705728000}+2\left(\frac{371153520}{6402373705728000}+\frac{1}{402361344000}\right) \\
& +\frac{230613840}{6402373705728000}+2\left(\frac{27123330}{6402373705728000}+\frac{1}{689762304000}\right) \\
& +\frac{9077796}{6402373705728000}+2\left(\frac{481338}{6402373705728000}+\frac{1}{1494484992000}\right) \\
& +\frac{48620}{6402373705728000}+\frac{2}{4184557977600}+\frac{2}{15692092416000} \\
& \left.+2(83691159552000)^{-1}+2(711374856192000)^{-1}+2(12804747411456000)^{-1}\right)
\end{aligned}
$$

Corollary 2.4 Let $k \in \mathbb{N}$ and $\alpha, p, L$ be positive real numbers, such that $L<1$ and $2^{p} \leq 2^{18} L$. Let $f: X \rightarrow Y$ be a mapping satisfying

$$
\left\|\operatorname{Df}\left(x_{1}, y_{1}\right), \ldots, \operatorname{Df}\left(x_{k}, y_{k}\right)\right\|_{k} \leq \alpha\left(\left\|x_{1}\right\|^{p}+\cdots+\left\|x_{k}\right\|^{p}+\left\|y_{1}\right\|^{p}+\cdots+\left\|y_{k}\right\|^{p}\right),
$$

for all $x_{1}, \ldots, x_{k}, y_{1}, \ldots, y_{k} \in X$. Then there exists a unique octadecic mapping $Q: X \rightarrow Y$, such that

$$
\left\|f\left(x_{1}\right)-Q\left(x_{1}\right), \ldots, f\left(x_{k}\right)-Q\left(x_{k}\right)\right\|_{k} \leq \frac{1}{2^{18}(1-L)} \psi\left(x_{1}, \ldots, x_{k}\right),
$$

for all $x_{1}, \ldots, x_{k} \in X$, where

$$
\begin{aligned}
\psi\left(x_{1}, \ldots, x_{k}\right):= & (3201186852864000)^{-1} \alpha\left(\left\|x_{1}\right\|^{p}+\cdots+\left\|x_{k}\right\|^{p}\right)(24310+87516 \\
& +31824\left(2^{p}+1\right)+18564\left(3^{p}+1\right)+8568\left(4^{p}+1\right)+3060\left(5^{p}+1\right)+816\left(6^{p}+1\right) \\
& +153\left(7^{p}+1\right)+18\left(8^{p}+1\right)+9^{p}+1+\frac{2^{p}}{2} \\
& +4\left(\frac{3234988548}{6402373705728000}+\frac{1}{2324754432000}+\frac{1}{355687428096000}\right) \\
& +4(2)^{p}\left(\frac{1922913180}{6402373705728000}+\frac{1}{41845579776000}+\frac{1}{292626432000}\right) \\
& +\frac{4(3)^{p} 9401058480}{6402373705728000}+4(4)^{p}\left(\frac{371153520}{6402373705728000}+\frac{1}{402361344000}\right) \\
& +\frac{4(5)^{p} 115306920}{6402373705728000}+4(6)^{p}\left(\frac{27123330}{6402373705728000}+\frac{1}{689762304000}\right) \\
& +\frac{4(7)^{p} 4538898}{6402373705728000}+4(8)^{p}\left(\frac{481338}{6402373705728000}+\frac{1}{1494484992000}\right) \\
& +\frac{4(9)^{p} 24310}{6402373705728000}+\frac{4(10)^{p}}{4184557977600}+\frac{4(12)^{p}}{15692092416000} \\
& +\frac{4(14)^{p}}{83691159552000}+\frac{4(16)^{p}}{711374856192000} \\
& \left.+4(18)^{p}(12804747411456000)^{-1}\right) .
\end{aligned}
$$

The following example shows that the assumption $2^{p} \leq 2^{18} L$ cannot be omitted in Corollary 2.4. We know from Example 1.2 that if

$$
\left\|x_{1}, \ldots, x_{k}\right\|_{k}=\sup \left\{\left|x_{1}\right|, \ldots,\left|x_{k}\right|\right\}
$$

then $\left(\left(\mathbb{R}^{k},\|\cdot\|_{k}\right): k \in \mathbb{N}\right)$ is a multi-normed space. 
Example 2.5 Let $k \in \mathbb{N}$. We define $\phi: \mathbb{R} \rightarrow \mathbb{R}$, by

$$
\phi(x):= \begin{cases}1 & x \in[1, \infty) \\ x^{18} & x \in(-1,1) \\ -1 & x \in(-\infty,-1] .\end{cases}
$$

We consider the function $f: \mathbb{R} \rightarrow \mathbb{R}$ defined by

$$
f(x)=\sum_{n=0}^{\infty} \frac{\phi\left(4^{n} x\right)}{4^{18 n}}, \quad(x \in \mathbb{R}) .
$$

Then $f$ satisfies the following functional inequality:

$$
\left\|\operatorname{Df}\left(x_{1}, y_{1}\right), \ldots, \operatorname{Df}\left(x_{k}, y_{k}\right)\right\|_{k} \leq \frac{2^{18}+18 !}{4^{18}-1} 4^{54}\left(\left|x_{1}\right|^{18}+\cdots+\left|x_{k}\right|^{18}+\left|y_{1}\right|^{18}+\cdots+\left|y_{k}\right|^{18}\right),
$$

for all $x_{1}, \ldots, x_{k}, y_{1}, \ldots, y_{k} \in \mathbb{R}$.

Proof We have

$$
|f(x)| \leq \frac{4^{18}}{4^{18}-1}
$$

for all $x \in \mathbb{R}$. Therefore, we see that $f$ is bounded. Let $x, y \in \mathbb{R}$. If $|x|^{18}+|y|^{18}=0$ or $|x|^{18}+|y|^{18} \geq \frac{1}{4^{18}}$, then

$$
|\operatorname{Df}(x, y)| \leq \frac{\left(2^{18}+18 !\right) 4^{18}}{4^{18}-1} \leq \frac{\left(2^{18}+18 !\right) 4^{18}}{4^{18}-1} 4^{18}\left(|x|^{18}+|y|^{18}\right) .
$$

Now, suppose that $0<|x|^{18}+|y|^{18}<\frac{1}{4^{18}}$. Then there exists a nonnegative integer $k$ such that

$$
\frac{1}{4^{18(k+2)}} \leq|x|^{18}+|y|^{18}<\frac{1}{4^{18(k+1)}} .
$$

Hence,

$$
4^{k} x<\frac{1}{4} \text { and } 4^{k} y<\frac{1}{4}
$$

and

$$
\begin{aligned}
& 4^{n}(x+9 y), 4^{n}(x+8 y), 4^{n}(x+7 y), 4^{n}(x+6 y), 4^{n}(x+5 y), 4^{n}(x+4 y), \\
& 4^{n}(x+3 y), 4^{n}(x+2 y), 4^{n}(x+y), 4^{n} x, 4^{n}(x-y), 4^{n}(x-2 y), 4^{n}(x-3 y), 4^{n}(x-4 y), \\
& 4^{n}(x-5 y), 4^{n}(x-6 y), 4^{n}(x-7 y), 4^{n}(x-8 y), 4^{n}(x-9 y), 4^{n} y \in(-1,1)
\end{aligned}
$$

for all $n=0,1, \ldots, k-1$. Thus we get

$$
\begin{aligned}
& \frac{|\operatorname{Df}(x, y)|}{|x|^{18}+|y|^{18}} \leq \sum_{n=k}^{\infty} \frac{2^{18}+18 !}{4^{18 n}\left(|x|^{18}+|y|^{18}\right)} \leq \sum_{n=0}^{\infty} \frac{2^{18}+18 !}{4^{18 n} 4^{18(k+2)}\left(|x|^{18}+\left|y^{18}\right|\right)} 4^{36} \\
& \leq \sum_{n=0}^{\infty} \frac{2^{18}+18 !}{4^{18 n}} 4^{36}=\frac{2^{18}+18 !}{4^{18}-1} 4^{54},
\end{aligned}
$$

or

$$
|\operatorname{Df}(x, y)| \leq \frac{2^{18}+18 !}{4^{18}-1} 4^{54}\left(|x|^{18}+|y|^{18}\right)
$$


Hence $f$ satisfies (2.1) for all $x_{1}, \ldots, x_{k}, y_{1}, \ldots, y_{k} \in \mathbb{R}$. Now, we claim that the octadecic functional equation (1.1) is not stable for $p=18$ in Corollary 2.4. Suppose on the contrary that there exists an octadecic mapping $C: \mathbb{R} \rightarrow \mathbb{R}$, such that

$$
\left\|f\left(x_{1}\right)-C\left(x_{1}\right), \ldots, f\left(x_{k}\right)-C\left(x_{k}\right)\right\|_{k} \leq \beta\left(\left|x_{1}\right|^{18}+\cdots+\left|x_{k}\right|^{18}\right)
$$

for some $\beta \in \mathbb{R}$ and all $x_{1}, \ldots, x_{k} \in \mathbb{R}$. So $|f(x)-C(x)| \leq \delta|x|^{18}$ for some constant $\delta>0$ and all $x \in \mathbb{R}$. Then there exists $\gamma \in \mathbb{R}$ for which $C(x)=\gamma x^{18}$ for all $x \in \mathbb{Q}$. Therefore,

$$
\frac{|f(x)|}{x^{18}} \leq \delta+|\gamma|, \quad(x \in \mathbb{Q}) .
$$

Let $M \in \mathbb{N}$ be such that $M>\delta+|\gamma|$. If $x$ is a rational number in $\left(0, \frac{1}{4^{M-1}}\right)$, then we have $4^{n} x \in(0,1)$ for each $n=0,1,2, \ldots, M-1$. Consequently, for such an $x$ we have

$$
\frac{f(x)}{x^{18}}=\sum_{n=0}^{\infty} \frac{\phi\left(4^{n} x\right)}{4^{18 n} x^{18}} \geq \sum_{n=0}^{M-1} \frac{4^{18 n} x^{18}}{4^{18 n} x^{18}}=M>\delta+|\gamma|,
$$

which leads to a contradiction.

Corollary 2.6 Let $k \in \mathbb{N}$ and $\alpha, p, L$ be positive real numbers, such that $L<1$ and $2^{2 k p} \leq 2^{18} L$. Let $f: X \rightarrow Y$ be a mapping satisfying

$$
\left\|\operatorname{Df}\left(x_{1}, y_{1}\right), \ldots, \operatorname{Df}\left(x_{k}, y_{k}\right)\right\|_{k} \leq \alpha\left\|x_{1}\right\|^{p} \cdots\left\|x_{k}\right\|^{p}\left\|y_{1}\right\|^{p} \cdots\left\|y_{k}\right\|^{p},
$$

for all $x_{1}, \ldots, x_{k}, y_{1}, \ldots, y_{k} \in X$. Then there exists a unique octadecic mapping $Q: X \rightarrow Y$, such that

$$
\left\|f\left(x_{1}\right)-Q\left(x_{1}\right), \ldots, f\left(x_{k}\right)-Q\left(x_{k}\right)\right\|_{k} \leq \frac{1}{2^{18}(1-L)} \psi\left(x_{1}, \ldots, x_{k}\right),
$$

for all $x_{1}, \ldots, x_{k} \in X$, where

$$
\begin{aligned}
\psi\left(x_{1}, \ldots, x_{k}\right):= & (3201186852864000)^{-1} \alpha\left\|x_{1}\right\|^{2 p} \ldots\left\|x_{k}\right\|^{2 p}\left(43758+31824(2)^{k p}\right. \\
& +18564(3)^{k p}+8568(4)^{k p}+3060(5)^{k p}+816(6)^{k p}+153(7)^{k p}+18(8)^{k p}+9^{k p} \\
& +2\left(\frac{3234988548}{6402373705728000}+\frac{1}{2324754432000}+\frac{1}{355687428096000}\right) \\
& +2(4)^{k p}\left(\frac{1922913180}{6402373705728000}+\frac{1}{41845579776000}+\frac{1}{292626432000}\right) \\
& +\frac{2(9)^{k p} 9401058480}{6402373705728000}+2(16)^{k p}\left(\frac{371153520}{6402373705728000}+\frac{1}{402361344000}\right) \\
& +\frac{2(25)^{k p} 115306920}{6402373705728000}+2(36)^{k p}\left(\frac{27123330}{6402373705728000}+\frac{1}{689762304000}\right) \\
& +\frac{2(49)^{k p} 4538898}{6402373705728000}+2(64)^{k p}\left(\frac{481338}{6402373705728000}+\frac{1}{1494484992000}\right) \\
& +\frac{2(81)^{k p} 24310}{6402373705728000}+\frac{2(100)^{k p}}{41845557977600}+\frac{2(144)^{k p}}{15692092416000} \\
& +\frac{2(196)^{k p}}{83691159552000}+\frac{2(256)^{k p}}{711374856192000} \\
& \left.+2(324)^{k p}(12804747411456000)^{-1}\right) .
\end{aligned}
$$

Corollary 2.7 Let $k \in \mathbb{N}$ and $\alpha, p, L$ be positive real numbers, such that $L<1$ and $4^{k p} \leq 2^{18} L$. Let $f: X \rightarrow Y$ be a mapping satisfying

$$
\begin{aligned}
& \left\|\operatorname{Df}\left(x_{1}, y_{1}\right), \ldots, \operatorname{Df}\left(x_{k}, y_{k}\right)\right\|_{k} \leq \alpha\left(\left\|x_{1}\right\|^{p} \ldots\left\|x_{k}\right\|^{p}\left\|y_{1}\right\|^{p} \ldots\left\|y_{k}\right\|^{p}\right. \\
& \left.+\left\|x_{1}\right\|^{2 k p}+\cdots+\left\|x_{k}\right\|^{2 k p}+\left\|y_{1}\right\|^{2 k p}+\cdots+\left\|y_{k}\right\|^{2 k p}\right)
\end{aligned}
$$


for all $x_{1}, \ldots, x_{k}, y_{1}, \ldots, y_{k} \in X$. Then there exists a unique octadecic mapping $Q: X \rightarrow Y$, such that

$$
\left\|f\left(x_{1}\right)-Q\left(x_{1}\right), \ldots, f\left(x_{k}\right)-Q\left(x_{k}\right)\right\|_{k} \leq \frac{1}{2^{18}(1-L)} \psi\left(x_{1}, \ldots, x_{k}\right),
$$

for all $x_{1}, \ldots, x_{k} \in X$, where

$$
\begin{aligned}
& \psi\left(x_{1}, \ldots, x_{k}\right):=(3201186852864000)^{-1} \alpha\left\|x_{1}\right\|^{2 p} \ldots\left\|x_{k}\right\|^{2 p}\left(43758+31824(2)^{k p}\right. \\
& +18564(3)^{k p}+8568(4)^{k p}+3060(5)^{k p}+816(6)^{k p}+153(7)^{k p}+18(8)^{k p}+9^{k p} \\
& +2\left(\frac{3234988548}{6402373705728000}+\frac{1}{2324754432000}+\frac{1}{355687428096000}\right) \\
& +2(4)^{k p}\left(\frac{1922913180}{6402373705728000}+\frac{1}{41845579776000}+\frac{1}{292626432000}\right) \\
& +\frac{2(9)^{k p} 9401058480}{6402373705728000}+2(16)^{k p}\left(\frac{371153520}{6402373705728000}+\frac{1}{402361344000}\right) \\
& +\frac{2(25)^{k p} 115306920}{6402373705728000}+2(36)^{k p}\left(\frac{27123330}{6402373705728000}+\frac{1}{689762304000}\right) \\
& +\frac{2(49)^{k p} 4538898}{6402373705728000}+2(64)^{k p}\left(\frac{481338}{6402373705728000}+\frac{1}{1494484992000}\right) \\
& +\frac{2(81)^{k p} 24310}{6402373705728000}+\frac{2(100)^{k p}}{4184557977600}+\frac{2(144)^{k p}}{15692092416000} \\
& +\frac{2(196)^{k p}}{83691159552000}+\frac{2(256)^{k p}}{711374856192000} \\
& \left.+2(324)^{k p}(12804747411456000)^{-1}\right) \\
& +(3201186852864000)^{-1} \alpha\left(\left\|x_{1}\right\|^{2 k p}+\ldots+\left\|x_{k}\right\|^{2 k p}\right)(24310+87516 \\
& +31824\left(4^{k p}+1\right)+18564\left(9^{k p}+1\right)+8568\left((16)^{k p}+1\right)+3060\left((25)^{k p}+1\right) \\
& +816\left((36)^{k p}+1\right)+153\left((49)^{k p}+1\right)+18\left((64)^{k p}+1\right)+(81)^{k p}+1+\frac{2^{2 k p}}{2} \\
& +4\left(\frac{3234988548}{6402373705728000}+\frac{1}{2324754432000}+\frac{1}{355687428096000}\right) \\
& +4(4)^{k p}\left(\frac{1922913180}{6402373705728000}+\frac{1}{41845579776000}+\frac{1}{292626432000}\right) \\
& +\frac{4(9)^{k p} 9401058480}{6402373705728000}+4(16)^{k p}\left(\frac{371153520}{6402373705728000}+\frac{1}{402361344000}\right) \\
& +\frac{4(25)^{k p} 115306920}{6402373705728000}+4(36)^{k p}\left(\frac{27123330}{6402373705728000}+\frac{1}{689762304000}\right) \\
& +\frac{4(49)^{k p} 4538898}{6402373705728000}+4(64)^{k p}\left(\frac{481338}{6402373705728000}+\frac{1}{1494484992000}\right) \\
& +\frac{4(81)^{k p} 24310}{6402373705728000}+\frac{4(100)^{k p}}{4184557977600}+\frac{4(144)^{k p}}{15692092416000} \\
& +\frac{4(196)^{k p}}{83691159552000}+\frac{4(256)^{k p}}{711374856192000} \\
& \left.+4(324)^{k p}(12804747411456000)^{-1}\right) \text {. }
\end{aligned}
$$

Open Access This article is distributed under the terms of the Creative Commons Attribution 4.0 International License (http:// creativecommons.org/licenses/by/4.0/), which permits unrestricted use, distribution, and reproduction in any medium, provided you give appropriate credit to the original author(s) and the source, provide a link to the Creative Commons license, and indicate if changes were made. 


\section{References}

1. Baker, J.: A general functional equation and its stability. Proc. Am. Math. Soc. 133, 1657-1664 (2005)

2. Czerwik, S.: Stability of Functional Equations of Ulam-Hyers-Rassias Type. Hadronic Press lnc, Florida (2003)

3. Dales, H.G.; Moslehian, M.S.: Stability of mapping on multi-normed spaces. Glasg. Math. J. 49, 321-332 (2007)

4. Dales, H.G.; Polyakov, M.E.: Multi-Normed Spaces and Multi-banach Algebras. University of Leeds, Leeds (2012)

5. Eshaghi Gordji, M.; Ghaemi, M.B.; Rassias, J.M.; Alizadeh, B.: Nearly ternary quadratic higher derivations on nonArchimedean ternary Banach algebras. A fixed point approach. Abstr. Appl. Anal. 2011, 1-18 (2011)

6. Eshaghi Gordji, M.; Cho, Y.J.; Ghaemi, M.B.; Majani, H.: Approximately quintic and sextic mappings form $r$-divisible groups into Sertnevic probabilistic Banach spaces. Fixed point method. Discrete Dyn. Nat. Soc. 5, 1-16 (2011)

7. Ghaemi, M.B.; Majani, H.; Eshaghi Gordji, M.: Approximately quintic and sextic mappings on the probabilistic normed spaces. Bull. Korean Math. Soc. 49, 339-352 (2012)

8. Hyers, D.H.: On the stability of the linear functional equation. Proc. Natl. Acad. Sci. USA 27, 222-224 (1941)

9. Moslehian, M.S.; Nikodem, K.; Popa, D.: Asymptotic aspect of the quadratic functional equation in multi-normed spaces. J. Math. Anal. Appl. 355, 717-724 (2009)

10. Nazarianpoor, M.; Rassias, J.M.; Sadeghi, Gh.: Stability and non stability of octadecic functional equation in Banach spaces (submitted)

11. Park, C.; Eshaghi Gordji, M.; Ghaemi, M.B.; Majani, H.: Fixed points and approximately octic mappings in non-Archimedean 2-normed spaces. J. Inequal. Appl. 2012, 11-12 (2012)

12. Rassias, J.M.: On approximation of approximately linear mappings by linear mappings. J. Funct. Anal. 46, 126-130 (1982)

13. Rassias, J.M.; Eslamian, M.: Fixed point and stability of nonic functional equation in quasi- $\beta$-normed spaces. Contemp. Anal. Appl. Math. 3, 293-309 (2015)

14. Rassias, J.M.; Kim, H.M.: Generalized Hyers-Ulam stability for general additive functional equation in quasi- $\beta$-normed spaces. J. Math. Anal. Appl. 356, 302-309 (2009)

15. Rassias, ThM: On the stability of the linear mapping in Banach spaces. Proc. Am. Math. Soc. 72, 297-300 (1978)

16. Ravi, K.; Rassias, J.M.; Pinelas, S.; Sabarinathan, S.: A fixed point approach to the stability of decic functional equation in quasi- $\beta$-normed spaces. Panam. Math. J. 25, 42-52 (2015)

17. Ulam, S.M.: A Collection of Mathematical Problems. Interscience Publishing, New York (1960)

18. Xu, T.; Rassias, J.M.: Approximate septic and octic mappings in quasi- $\beta$-normed spaces. J. Comput. Anal. Appl. 15, 1110 1119 (2013)

19. Xu, T.; Rassias, J.M.; Xu, W.X.: A fixed point approach to the stability of a general mixed type additive-cubic functional equation in quasi fuzzy normed spaces. Int. J. Phys. Sci. 6, 313-324 (2011)

20. Xu, T.; Rassias, J.M.; Xu, W.X.: A fixed point approach to the stability of quintic and sextic functional equations in quasi$\beta$-normed spaces. J. Inequal. Appl. 2010, 1-23 (2010)

Publisher's Note Springer Nature remains neutral with regard to jurisdictional claims in published maps and institutional affiliations. 